\title{
SIGPLAN
}

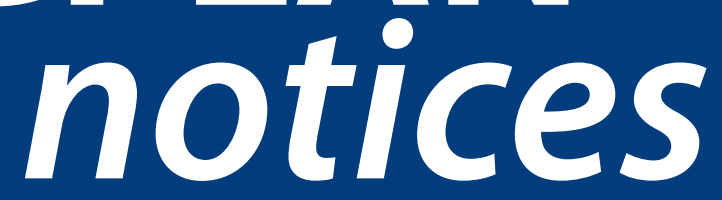

Proceedings of the 37th ACM SIGPLAN Conference on

\section{Programming Language}

\section{Design and Implementation (PLDI'16)}


ACM SIGPLAN Notices

A monthly publication of ACM SIGPLAN

\section{Publications Office}

ACM, 2 Penn Plaza, Suite 701, New York, NY 10121-0701, USA

$+1-212-869-7440$

$F A X+1-212-302-9618$

ACM SIGPLAN Notices is an informal monthly publication of the Special Interest Group on Programming Languages (SIGPLAN) of ACM.

Membership in SIGPLAN is open to ACM Members or associate members for $\$ 25$ (online) and $\$ 130$ (print). Membership for ACM Student members is $\$ 15$ (online) and $\$ 130$ (print). SIGPLAN Notices is available online through the ACM Digital Library. Print members receive a hardcopy of each issue. All members receive the annual SIGPLAN CD, discounts at SIGPLAN events, and may vote in the Group's elections. ACM members of SIGPLAN may serve as officers of the Group.

Institutional or Library subscriptions to ACM SIGPLAN Notices are available for $\$ 110$ per year, and the regular back issues of the Notices may be purchased for $\$ 19$ per copy from ACM Headquarters.

Periodicals postage paid at New York, NY 10001 and at additional mailing offices.

Requests for reprints, copies of reports, or references should be sent to authors.

\section{Members Services Information: acmhelp@acm.org \\ or +1-212-626-0500}

\section{Change of address:} acmcoa@acm.org

\section{Advertising Information:} Jennifer Ruzicka +1-212-869-7440

POSTMASTER: Send change of Address to ACM SIGPLAN Notices, ACM, 2 Penn Plaza, Suite 701, New York, NY 10121-0701.
SIGPLAN Executive Committee

\section{Chair}

Michael Hicks

University of Maryland

mwh@cs.umd.edu

\section{Vice Chair}

Peter Thiemann

Universität Freiburg

thiemann@informatik.uni-freiburg.de

\section{Treasurer}

Cristina Lopes

University of California, Irvine

lopes@ics.uci.edu

\section{Secretary}

Kathryn S. McKinley

Microsoft Research

mckinley@microsoft.com

\section{Members at Large}

Emery Berger

University of Massachusetts Amherst emery@cs.umass.edu

Susan Eisenbach

Imperial College, London

s.eisenbach@imperial.ac.uk

Gabriele Keller

University of New South Wales

keller@cse.unsw.edu.au

\section{Satnam Singh \\ Facebook \\ s.singh@acm.org}

Yannis Smaragdakis

University of Athens

smaragd@di.uoa.gr

\section{Past Chair}

Jan Vitek

Northeastern University

j.vitek@neu.edu

Past Vice Chair

Jeremy Gibbons

University of Oxford

jeremy.gibbons@cs.ox.ac.uk

\section{Information Director}

Matthew Fluet

Rochester Institute of Technology

mtf@cs.rit.edu

\section{SIGPLAN Program Director \\ Fran Spinola spinola@hq.acm.org}

\section{SIGPLAN Sponsored Conferences}

Architectural Support for Programming Languages and Operating

Systems (ASPLOS)

Code Generation and

Optimization (CGO)

Dynamic Languages Symposium (DLS)

Generative Programming: Concepts and Experiences (GPCE)

Haskell Symposium (HS)

International Conference on Functional Programming (ICFP)

International Symposium on Memory Management (ISMM)

Languages, Compilers, and Tools for Embedded Systems (LCTES)

Object-oriented Programming, Systems, Languages, and Applications (OOPSLA)

Onward!

Principles and Practices of Parallel Programming (PPOPP)

Principles of Programming Languages (POPL)

Programming Language Design and Implementation (PLDI)

Software Language Engineering (SLE)

Systems, Programming, Languages and Applications: Software for Humanity (SPLASH)

Virtual Execution Environments (VEE)

\author{
SIGPLAN Online \\ sigplan.org \\ facebook.com/SIGPLAN \\ twitter.com/sigplan
}

\section{SIGPLAN Mission Statement}

SIGPLAN, the premier programming languages organization, promotes awareness and advancement of research and practice in programming languages. SIGPLAN accomplishes its mission by sponsoring conferences and publishing newsletters. The SIG also serves as a coordinating body for the volunteers who wish to undertake projects to further the goals of the SIG. 
Association for

Computing Machinery

June 13-17, 2016

Santa Barbara, CA, USA

Advancing Computing as a Science \& Profession

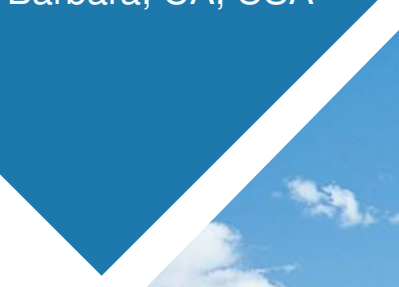

\section{PLDI'16}

Proceedings of the 37th ACM SIGPLAN Conference on

\section{Programming Language Design and Implementation}

Edited by:

Chandra Krintz and Emery Berger

Sponsored by:

ACM SIGPLAN

Supported by:

NSF, Facebook, Intel, Oracle Labs, Google, Grammatech, HP, Huawei, IBM Research, Microsoft Research 


\section{The Association for Computing Machinery, Inc. 2 Penn Plaza, Suite 701 New York, NY 10121-0701}

Copyright (c) 2016 by the Association for Computing Machinery, Inc (ACM). Permission to make digital or hard copies of portions of this work for personal or classroom use is granted without fee provided that the copies are not made or distributed for profit or commercial advantage and that copies bear this notice and the full citation on the first page. Copyrights for components of this work owned by others than ACM must be honored. Abstracting with credit is permitted.

To copy otherwise, to republish, to post on servers or to redistribute to lists, requires prior specific permission and/or a fee. Request permission to republish from: Publications Dept. ACM, Inc.

Fax +1-212-869-0481 or E-mail permissions@acm.org.

For other copying of articles that carry a code at the bottom of the first or last page, copying is permitted provided that the per-copy fee indicated in the code is paid through the Copyright Clearance Center, 222 Rosewood Drive, Danvers, MA 01923.

\section{Notice to Past Authors of ACM-Published Articles}

ACM intends to create a complete electronic archive of all articles and/or other material previously published by ACM. If you have written a work that was previously published by ACM in any journal or conference proceedings prior to 1978, or any SIG Newsletter at any time, and you do NOT want this work to appear in the ACM Digital Library, please inform permissions@acm.org, stating the title of the work, the author(s), and where and when published.

ACM ISBN: 978-1-4503-4261-2

Additional copies may be ordered prepaid from:

$\begin{array}{ll}\text { ACM Order Department } & \text { (U.S.A. and Canada) } \\ \text { P.O. BOX 11405 } & +1-212-626-0500 \\ \text { Church Street Station } & \text { (All other countries) } \\ \text { New York, NY 10286-1405 } & \text { Fax: +1-212-944-1318 } \\ & \text { E-mail: acmhelp@ acm.org }\end{array}$

Cover photo from

http: //santabarbaraca.com/

Production: Conference Publishing Consulting

D-94034 Passau, Germany, info@conference-publishing.com 


\section{Message from the Chairs}

Welcome to PLDI 2016, the 37th ACM SIGPLAN Conference on Programming Language Design and Implementation; held this year in Santa Barbara, California. PLDI is the premier research conference on programming languages and their implementation.

This year, there were 304 papers submitted to PLDI -- a record, just beating last year's 303. A number of papers unfortunately violated the formatting or double blind rules and were not considered; the technical program committee ended up reviewing 288 papers. Of these, 48 were accepted for inclusion in the 2016 program. Two papers submitted to TOPLAS were also accepted to appear at PLDI.

This year, we incorporated a number of changes, all aimed at improving the quality and integrity of the process; many of these approaches are time-tested in other communities. In addition to the Program Committee (PC), we added an External Program Committee (EPC) consisting of senior members of the PL community who acted as a "light" program committee; their charge was to review roughly ten papers each, and in particular, to review all PC-authored papers. The EPC met via a day-long teleconference prior to the physical PC meeting. A subset of the EPC (Vikram Adve, Rastislav Bodik, Mike Ernst, Cormac Flanagan, and Manu Sridharan) formed the Distinguished Papers Committee, whose job it was to select the distinguished papers via a conference-like process. The committee selected four Distinguished Paper Awards, which will be announced at the conference. We also changed the way the External Review Committee (ERC) was used. Rather than using the ERC to shed load, as has been done in the past, the ERC specifically this year acted as an expertise pool. We also expanded the membership pool of the ERC to include senior and graduating PhD students; these students were nominated and vetted by the PC and EPC. These students were asked to review papers directly related to their thesis work; the quality of the resulting reviews was indistinguishable from the best reviews provided by PC and EPC members.

Most of the technical program committee members provided excellent, in-depth reviews. The committee was asked to ensure that their reviews were at least 500 words long (an automatic option available in HotCRP) and most reviewers went above and beyond this request. We checked that all reviews provided the appropriate level of detail, gave solid, actionable feedback to authors, and struck the right tone. In addition, reviewers were directed to read and respond to author responses, and a summary of the (often quite extensive) on-line and PC meeting discussions was included with all papers to provide as much feedback as possible to the authors. We hope that the stellar level of engagement and the quality and depth of reviews provided by the committee members sets a benchmark for future conferences.

To ensure that conflicts of interest were handled properly (and early), all committee members were asked to vet any conflicts declared by authors that they had not previously declared. Rather than using traditional bidding, we adopted a "split preference-expertise" approach that has been used by POPL in the past: bidding on all papers was split between a preference grade and an expertise grade (for example, 3X), which HotCRP now supports. This approach helped 
to ensure a good match of high expertise to papers. We modified the review form itself to include very precise directions, and added several fields that helped us ensure that papers received as high expertise reviews as possible and to promote productive discussion on-line: (1) a formal explanation of expertise and confidence, (2) an expertise field for reviewers to explain their specific expertise on each paper, which helped us cover expertise gaps, and (3) a championship field for reviewers to explain to other reviewers their reasons for championing a particular paper. We employed a double-blind reviewing process; during reviewing, authorship was only disclosed when deemed absolutely necessary (this happened very rarely), and in general, authorship was only disclosed for accepted papers. This approach avoids implicit bias and prevents compromising future double blind reviewing for rejected papers, which comprise the majority of submitted papers. A robust, on-line discussion led to consensus on most papers before the physical meeting held in Saint Petersburg, Florida. This freed the program committee to focus on contentious papers and to resolve technical questions in an in-person, high bandwidth setting. Finally, we introduced shepherding for all papers to make it easier to accept papers with minor flaws. All but one paper successfully completed the shepherding process, and the consensus was that the process resulted in substantially improved papers.

We thank all of the committee members and expert external reviewers for their hard work and careful reviews, past and future program chairs and others who provided valuable feedback (especially Martin Vechev, Yannis Smaragdakis, Steve Freund, and Eran Yahav), and Eddie Kohler, who promptly fixed several bugs in HotCRP and added some key features that should improve the lives of future program chairs.

The organization of the PLDI conference is the result of the combined efforts of an amazing group of individuals. We especially thank Annabel Satin (ACM SIGPLAN Conference Manager), the ACM Conference support staff, all of our wonderful student volunteers, and the outstanding members of the PLDI16 Organizing Committee: Ben Hardekopf (Student Volunteer and Student Research Competition Chair), Iulian Neamtiu (Posters Chair), John Regehr and Zachary Tatlock (Artifact Evaluation Co-Chairs), Tiark Rompf (Sponsorship Chair), Manu Sridharan (Publicity Chair), and Xiangyu Zhang (Workshops and Tutorials Chair). We thank David Grove (PLDI15 General Chair), Jan Vitek (Past ACM SIGPLAN Chair and PLDI Steering Committee Chair), Mike Hicks (Current ACM SIGPLAN Chair), Conference Publishing, everyone involved in the local arrangements in Santa Barbara, and the PLDI Steering Committee for their help, support, and guidance. Finally, we thank the authors, keynote speakers, and presenters of the conference and its co-located workshops, tutorials, and conferences for helping to make this an engaging and interesting event for our community. We hope you enjoy the conference!

Dr. Chandra Krintz

PLDI'16 General Chair

Univ. of California, Santa Barbara
Dr. Emery Berger

PLDI'16 Program Chair

Univ. of Massachusetts, Amherst 


\section{PLDI 2016 Organization}

\section{Organizing Committee}

\section{General Chair}

Chandra Krintz

Program Chair

Emery Berger
Univ. of California, Santa Barbara, USA

Univ. of Massachusetts, Amherst, USA

\section{Artifact Evaluation Co-Chairs}

John Regehr

Univ. of Utah, USA

Zachary Tatlock

Univ. of Washington, USA

Workshop and Tutorials Chair

Xiangyu Zhang

Purdue Univ., USA

Sponsorship Chair

Tiark Rompf

Purdue Univ. and Oracle Labs, USA

Publicity Chair

Manu Sridharan

Samsung Research America, USA

Student Volunteers and Research Competition Chair

Ben Hardekopf

Univ. of California, Santa Barbara, USA

Event Manager

Annabel Satin

PCK, London, UK

Steering Committee

Steve Blackburn

Hans-J. Boehm

Kathleen Fisher

Cormac Flanagan

Jeremy Gibbons

David Grove

Mike Hicks

Michael O'Boyle

Keshav Pingali

Peter Thiemann

Jan Vitek (Chair)

Philip Wadler

Australian National Univ., Australia

Google, USA

Tufts University, USA

Univ. of California, Santa Cruz, USA

Univ. of Oxford, UK

IBM Research, USA

Univ. of Maryland, USA

Univ. of Edinburgh, UK

Univ. of Texas, Austin, USA

Universität Freiburg, Germany

Northeastern Univ., USA

Univ. of Edinburgh, UK 


\section{Program Committee}

Martha Kim, Columbia Univ., USA

Hans Boehm, Google, USA

David Brumley, Carnegie Mellon Univ., USA

Cristian Cadar, Imperial College London, UK

Michael Carbin, MIT, USA

Albert Cohen, INRIA, France

Charlie Curtsinger, Grinnell College, USA

Loris D'Antoni, Univ. of Wisconsin-Madison, USA

Isil Dillig, Univ. of Texas, Austin, USA

Bryan Ford, EPFL, Switzerland

Matt Fredrikson, Carnegie Mellon Univ., USA

Patrice Godefroid, Microsoft Research, USA

Arjun Guha, Univ. of Massachusetts, Amherst, USA

Sam Guyer, Tufts Univ., USA

Manuel Hermenegildo, IMDEA Software Institute and T.U. of Madrid, Spain

Michael Hicks, Univ. of Maryland at College Park, USA

Tony Hosking, Purdue Univ., USA

Ranjit Jhala, Univ. of California, San Diego, USA

Brandon Lucia, Carnegie Mellon Univ., USA

Stephen McCamant, Univ. of Minnesota, USA

Todd Millstein, Univ. of California, Los Angeles, USA

Todd Mytkowicz, Microsoft Research, USA

Santosh Nagarakatte, Rutgers Univ., USA

Guilherme Ottoni, Facebook, USA

Cindy Rubio-Gonzalez, Univ. of California, Davis, USA

Caitlin Sadowski, Google, USA

Adrian Sampson, Cornell Univ. \& Microsoft Research, USA

Yannis Smaragdakis, Univ. of Athens, Greece

Zhendong Su, Univ. of California, Davis, USA

Zachary Tatlock, Univ. of Washington, USA

Martin Vechev, ETH Zurich, Switzerland

Eran Yahav, Technion, Isreal

Jean Yang, Carnegie Mellon Univ., USA

Greta Yorsh, Queen Mary Univ. of London, UK 


\section{External Program Committee}

Vikram Adve, Univ. of Illinois at Urbana-Champaign, USA

Steve Blackburn, Australian National Univ., Australia

Rastislav Bodik, Univ. of Washington, USA

Swarat Chaudhuri, Rice Univ., USA

Michael D. Ernst, Univ. of Washington, USA

Cormac Flanagan, Univ. of California, Santa Cruz, USA

Robert Grimm, Goldman Sachs, USA

Michael Hind, IBM Research, USA

Julia Lawall, LIP6, USA

Doug Lea, State Univ. of New York Oswego, USA

Ben Liblit, Univ. of Wisconsin-Madison, USA

Kathryn S. McKinley, Microsoft Research, USA

Michelle Mills Strout, Colorado State Univ., USA

Andrew Myers, Cornell Univ., USA

Robert O'Callahan, Mozilla Corporation, USA

Simon Peyton Jones, Microsoft Research, Cambridge, UK

Keshav Pingali, Univ. of Texas, Austin, USA

Martin Rinard, MIT, USA

Mooly Sagiv, Tel Aviv Univ., Isreal

Armando Solar-Lezama, MIT, USA

Manu Sridharan, Samsung Research America, USA

Guy L. Steele Jr., Oracle Labs, USA

David Walker, Princeton Univ., USA

Benjamin Zorn, Microsoft Research, USA

\section{Distinguished Paper Committee}

Vikram Adve, Univ. of Illinois at Urbana-Champaign, USA

Emery Berger, Univ. of Massachusetts, Amherst, USA

Rastislav Bodik, Univ. of Washington, USA

Michael D. Ernst, Univ. of Washington, USA

Cormac Flanagan, Univ. of California, Santa Cruz, USA

Manu Sridharan, Samsung Research America, USA 


\section{External Review Committee}

Eddie Aftandilian, Google, USA

Aws Albarghouthi, Univ. of Wisconsin, USA

Owen Arden, Cornell Univ., USA

George Balatsouras, Univ. of Athens, Greece

Dan Barowy, Univ. of Massachusetts, Amherst, USA

Mark Batty, Univ. of Cambridge, USA

Robert Bocchino, NASA/Caltech Jet Propulsion Laboratory, USA

Perry Cheng, IBM Research, USA

Ravi Chugh, Univ. of Chicago, USA

John Criswell, Univ. of Rochester, USA

Eva Darulova, MPI-SWS, Germany

George Fourtounis, Univ. of Athens, Greece

Sicun Gao, MIT, USA

Joe Gibbs-Politz, Swarthmore College, USA

Colin Gordon, Drexel Univ., USA

William Harris, Georgia Tech, USA

Hank Hoffman, Univ. of Chicago, USA

Justin Hsu, Univ. of Pennsylvania, USA

Melanie Kambadur, Columbia Univ., USA

Zachary Kincaid, Univ. of Toronto, Canada

Murali Krishna Ramanathan, Indian Institute of Science, Bangalore, India

Vu Le, Microsoft, USA

Jay Ligatti, Univ. of South Florida, USA

Jed Liu, Cornell Univ., USA

Fan Long, MIT, USA

Dan Lustig, Princeton Univ./NVIDIA, USA

Saeed Maleki, Microsoft Research, USA

Daniel Marino, Symantec Research Labs, USA

Sasa Misailovic, ETH Zurich, Switzerland

Peter Ohmann, Univ. of Wisconsin, USA

Peter-Michael Osera, Grinnell College, USA

Marco Pistoia, IBM Research, USA

Michael Pradel, TU Darmstadt, Germany

Rodric Rabbah, IBM Research, USA

Arjun Radhakrishna, Univ. of Pennsylvania, USA

Cosmin Radoi, Univ. of Illinois, USA 
Jonathan Ragan-Kelley, Stanford, USA

Mukund Raghotaman, Univ. of Pennsylvania, USA

Aseem Rastogi, Univ. of Maryland, College Park, USA

Veselin Raychev, ETH Zurich, Switzerland

Cole Schlesinger, Samsung Research America, USA

Julian Shun, Univ. of California, Berkeley, USA

Stelios Sidiroglou-Douskos, MIT, USA

Robert Soulé, Universita della Svizzera italiana (USI), Italy

Gordon Stewart, Ohio Univ., USA

Aditya Thakur, Google, USA

Emma Tosch, Univ. of Massachusetts Amherst, USA

Vlad Ureche, EPFL, Switzerland

John Vilk, Univ. of Massachusetts, Amherst, USA

Bo Wu, Colorado School of Mines, USA

Xi Yang, Australian National Univ., Australia

\section{Artifact Evaluation Committee}

Mehrdad Afshari, Univ. of California, Davis, USA

Spenser Bauman, Indiana Univ., USA

Joshua Cranmer, Univ. of Illinois at Urbana-Champaign, USA

Monika Dhok, Indian Institute of Science, Bangalore, India

Will Dietz, Univ. of Illinois at Urbana-Champaign, USA

Marko Dimjašević, Univ. of Utah, USA

Dana Drachsler Cohen, Technion, Isreal

Jean-Baptiste Jeannin, Carnegie Mellon Univ., USA

Xiangqi Li, Univ. of Utah, USA

Petr Maj, ReactorLabs, USA

David Menendez, Rutgers Univ., USA

Nhat Minh Le, ENS Paris, France

José Morales, IMDEA Software Institute, Spain

Justin Pombrio, Brown Univ., USA

Chenxiong Qian, Georgia Tech, USA

John Regehr, Univ. of Utah, USA

Malavika Samak, Indian Institute of Science, Bangalore, India

Vilhelm Sjoberg, Yale Univ., USA

Chengnian Sun, Univ. of California at Davis, USA 
Jubi Taneja, Univ. of Utah, USA

Niki Vazou, Univ. of California, San Diego, USA

Shiyi Wei, Virginia Tech, USA

James R. Wilcox, Univ. of Washington, USA

Doug Woos, Univ. of Washington, USA

Adarsh Yoga, Rutgers Univ., USA 


\section{PLDI'16 Keynote Presentations}

\section{Keynote \#1: Dr. Benjamin Zorn, Microsoft Research}

Title: Programming Languages and Technical Disruption

Abstract: What do cheating on fuel economy, the London Whale, and building a better mosquito trap have in common?

We are constantly bombarded with technical innovations that disrupt business models, social structures, labor markets, etc. Widely visible technical advances such as Internet of Things, Big Data, and Deep Learning are driving markets and creating a huge demand for computer science education. What role, if any, does programming language research have in an age of technical disruptions? In my talk, I argue that historically, as well as today, programming languages are central to major technical disruptions and that language innovation is often driven by technical innovation in other areas. To have the most impact, language researchers have a great opportunity to look beyond problems in their own research area to embrace and understand the impact that their ideas can have on critical societal problems. Increasing, people are assuming that software will be an essential part of solutions to societal problems. At the same time we know that building an infrastructure on software creates new challenges that threaten to reduce or eliminate the benefits altogether. To make the discussion concrete, I consider three problem domains: global health, financial market stability, and cybersecurity. In each case, I argue that programming language research can and should have a lot of impact. My challenge to the audience is to embrace these problems enthusiastically and bring the great depth of insight and innovation that the field has already created to the broadest audience possible.

Since I will be discussing topics that I have far too limited knowledge of, consider this a great opportunity to hear me say outrageous things that are almost certainly not true but at the same time hopefully provocative and entertaining.

Brief Biography: Ben Zorn is a Research Manager and Principal Researcher, co-managing the Research in Software Engineering (RiSE) group, a group of over 30 researchers and developers working on programming languages and software engineering in Microsoft Research, Redmond. After receiving a PhD in Computer Science from UC Berkeley in 1989, he served eight years on the Computer Science faculty at the University of Colorado in Boulder, receiving tenure and being promoted to Associate Professor in 1996. Dr. Zorn left the University of Colorado in 1998 to join Microsoft Research, where he currently works. His research interests include programming language design and implementation for reliability, security, and performance. He has served as both Program Chair (1999) and General Chair (2010) of the PLDI conference, as an Associate Editor of the ACM journals Transactions on Programming Languages and Systems and Transactions on Architecture and Code Optimization. He has also served seven years as a Member-at-Large of the SIGPLAN Executive Committee and four years as a member of the ACM Software Systems Award Committee. He is currently a member of the Computing Community Consortium (CCC) Council, a committee of the Computing

Research Association. For more information, visit his web page at: http://research.microsoft.com/ zorn/. 


\section{Keynote \#2: Dr. Luiz André Barroso}

Title: Programming a Warehouse-Scale Computer

Abstract: Public clouds are quickly making massive-scale computing capabilities available to an ever larger population of programmers, and are no longer a playground restricted to a handful of institutions, such as national labs or large Internet services companies. In this talk, we will highlight some of the features of this new class of computers, the challenges faced by their programmers, and the tools and techniques that we have developed to address some of those challenges.

Brief Biography: I am a Google Fellow, and the VP of Engineering for the Geo Platform team, the group responsible for collecting and curating maps, local and imagery data that powers our consumer products (such as Google Search and Google Maps). My technical interests that range from distributed systems software to the design of Google's computing platform. While at Google I have co-authored some well-cited articles on warehouse-scale computing, energy proportionality and storage system reliability. I also co-wrote "The Datacenter as a Computer", the first textbook to describe the architecture of warehouse-scale computing systems, now in its 2nd edition. I was previously a member of the research staff at Digital Equipment Corporation and Compaq, where our group did some of the pioneering research on modern multi-core architectures. Some of those multi-core processors also use variants of the ring-based cachecoherency interconnects that were the subject of my doctoral research.

I am a Fellow of the Association for Computing Machinery (ACM) and the American Association for the Advancement of Science. I was the program chair of ACM ISCA'09, a keynote speaker at FCRC'11, SIGMOD'10, ASPLOS'09, and a National Academy of Engineering Gilbreth Lectureship awardee in 2012. I am currently serving at the National Academies' Computer Science and Telecommunications Board. I hold B.S. and M.S. degrees in Electrical Engineering from the Pontifícia Universidade Católica of Rio de Janeiro, and a Ph.D. in Computer Engineering from the University of Southern California. 


\section{Sponsors and Supporters}

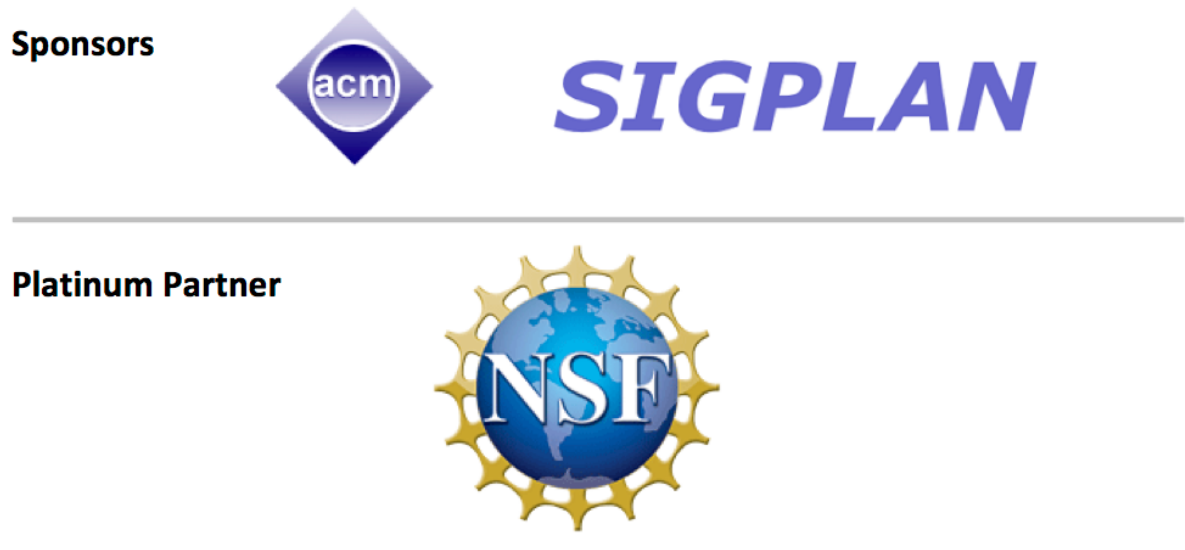

Gold partners

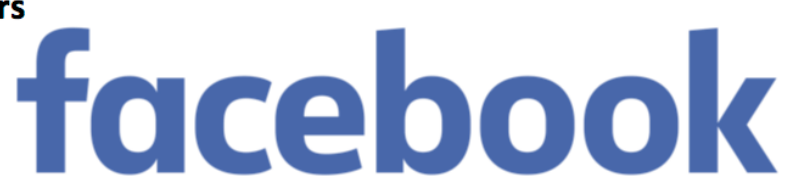

Silver partners

Oracle Labs

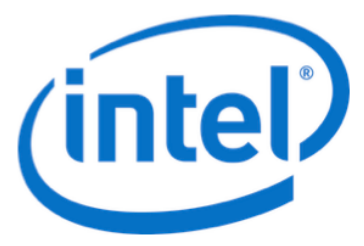

Bronze partners

Microsoft $\underset{\text { Huawel }}{\mathbb{N}}$ Research
Research Google (9) grammatech 


\section{Contents}

\section{Frontmatter}

Message from the Chairs $\ldots \ldots \ldots \ldots \ldots \ldots \ldots \ldots \ldots \ldots \ldots$ iii

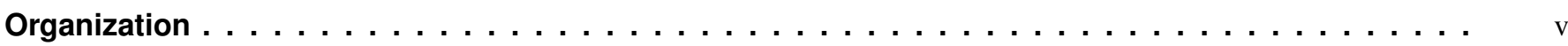

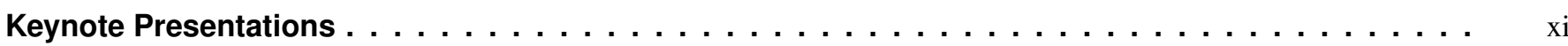

Sponsors and Supporters $\ldots \ldots \ldots \ldots \ldots \ldots \ldots \ldots \ldots \ldots \ldots \ldots \ldots \ldots \ldots \ldots \ldots$

Research Papers

\section{Down to the Metal I}

Into the Depths of C: Elaborating the De Facto Standards

Kayvan Memarian, Justus Matthiesen, James Lingard, Kyndylan Nienhuis, David Chisnall, Robert N. M. Watson, and

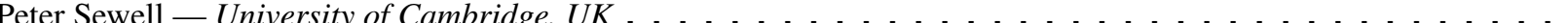

Living on the Edge: Rapid-Toggling Probes with Cross-Modification on x86

Buddhika Chamith, Bo Joel Svensson, Luke Dalessandro, and Ryan R. Newton - Indiana University, USA . . . . .

Polymorphic Type Inference for Machine Code

Matt Noonan, Alexey Loginov, and David Cok — GrammaTech, USA . . . . . . . . . . . . . . .

\section{Verification I}

\section{Data-Driven Precondition Inference with Learned Features}

Saswat Padhi, Rahul Sharma, and Todd Millstein - University of California at Los Angeles, USA; Stanford University,

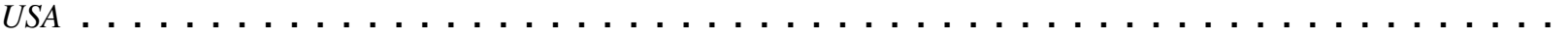

Cartesian Hoare Logic for Verifying k-Safety Properties

Marcelo Sousa and Isil Dillig - University of Oxford, UK; University of Texas at Austin, USA . . . . . . . . . . .

Verifying Bit-Manipulations of Floating-Point

Wonyeol Lee, Rahul Sharma, and Alex Aiken — Stanford University, USA ～. . . . . . . . . . . . .

\section{Testing and Debugging}

Coverage-Directed Differential Testing of JVM Implementations

Yuting Chen, Ting Su, Chengnian Sun, Zhendong Su, and Jianjun Zhao — Shanghai Jiao Tong University, China; East China Normal University, China; University of California at Davis, USA; Shanghai Jiao Tong University, Japan . .

Exposing Errors Related to Weak Memory in GPU Applications

Tyler Sorensen and Alastair F. Donaldson — Imperial College London, UK . . . . . . . . . . . . . .

Lightweight Computation Tree Tracing for Lazy Functional Languages

Maarten Faddegon and Olaf Chitil - University of Kent, UK . . . . . . . . . . . . . . . . . . .

\section{Energy and Performance}

\section{Effective Padding of Multidimensional Arrays to Avoid Cache Conflict Misses}

Changwan Hong, Wenlei Bao, Albert Cohen, Sriram Krishnamoorthy, Louis-Noël Pouchet, Fabrice Rastello, J. Ramanujam, and P. Sadayappan — Ohio State University, USA; Inria, France; Pacific Northwest National Laboratory, USA;

Louisiana State University, USA . . . . . . . . . . . . . . . . . . . . . . . . . . . . . . . .

GreenWeb: Language Extensions for Energy-Efficient Mobile Web Computing

Yuhao Zhu and Vijay Janapa Reddi - University of Texas at Austin, USA . . . . . . . . . . . . . . .

Input Responsiveness: Using Canary Inputs to Dynamically Steer Approximation

Michael A. Laurenzano, Parker Hill, Mehrzad Samadi, Scott Mahlke, Jason Mars, and Lingjia Tang - University of

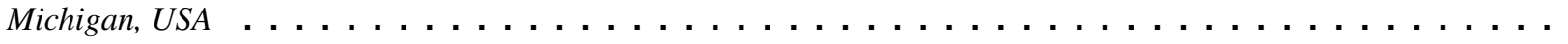




\section{New Languages}

\section{Configuration Synthesis for Programmable Analog Devices with Arco}

Sara Achour, Rahul Sarpeshkar, and Martin C. Rinard - Massachusetts Institute of Technology, USA; Dartmouth College,

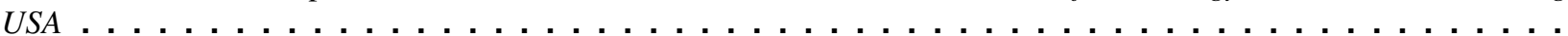

From Datalog to Flix: A Declarative Language for Fixed Points on Lattices

Magnus Madsen, Ming-Ho Yee, and Onď̌ej Lhoták — University of Waterloo, Canada . . . . . . . . . . . .

Latte: A Language, Compiler, and Runtime for Elegant and Efficient Deep Neural Networks

Leonard Truong, Rajkishore Barik, Ehsan Totoni, Hai Liu, Chick Markley, Armando Fox, and Tatiana Shpeisman — Intel

Labs, USA; University of California at Berkeley, USA . . . . . . . . . . . . . . . . . . . . . .

\section{Parsing and Compilation}

On the Complexity and Performance of Parsing with Derivatives

Michael D. Adams, Celeste Hollenbeck, and Matthew Might - University of Utah, USA . . . . . . . . . . . 224

\section{Down to the Metal II}

Stratified Synthesis: Automatically Learning the x86-64 Instruction Set

Stefan Heule, Eric Schkufza, Rahul Sharma, and Alex Aiken — Stanford University, USA; VMware, USA . . . . .

Remix: Online Detection and Repair of Cache Contention for the JVM

Ariel Eizenberg, Shiliang Hu, Gilles Pokam, and Joseph Devietti — University of Pennsylvania, USA; Intel, USA . .

Statistical Similarity of Binaries

Yaniv David, Nimrod Partush, and Eran Yahav — Technion, Israel ～. . . . . . . . . . . . . . . . . 266

\section{Types I}

\section{Accepting Blame for Safe Tunneled Exceptions}

Yizhou Zhang, Guido Salvaneschi, Quinn Beightol, Barbara Liskov, and Andrew C. Myers - Cornell University, USA;

TU Darmstadt, Germany; Massachusetts Institute of Technology, USA ． . . . . . . . . . . . . . . . .

Occurrence Typing Modulo Theories

Andrew M. Kent, David Kempe, and Sam Tobin-Hochstadt — Indiana University, USA . . . . . . . . . . .

Refinement Types for TypeScript

Panagiotis Vekris, Benjamin Cosman, and Ranjit Jhala - University of California at San Diego, USA . . . . . . .

\section{Synthesis I}

\section{MapReduce Program Synthesis}

Calvin Smith and Aws Albarghouthi - University of Wisconsin-Madison, USA . . . . . . . . . . . . . 326

Programmatic and Direct Manipulation, Together at Last

Ravi Chugh, Brian Hempel, Mitchell Spradlin, and Jacob Albers - University of Chicago, USA . . . . . . . . 341

Fast Synthesis of Fast Collections

Calvin Loncaric, Emina Torlak, and Michael D. Ernst — University of Washington, USA . . . . . . . . . . 355

\section{Software-Defined Networking}

\section{Event-Driven Network Programming}

Jedidiah McClurg, Hossein Hojjat, Nate Foster, and Pavol Černý - University of Colorado at Boulder, USA; Cornell

University, USA . . . . . . . . . . . . . . . . . . . . .

Temporal NetKAT

Ryan Beckett, Michael Greenberg, and David Walker - Princeton University, USA; Pomona College, USA . . . . 386

SDNRacer: Concurrency Analysis for Software-Defined Networks

Ahmed El-Hassany, Jeremie Miserez, Pavol Bielik, Laurent Vanbever, and Martin Vechev — ETH Zurich, Switzerland 


\section{Verifying Systems}

\section{Rehearsal: A Configuration Verification Tool for Puppet}

Rian Shambaugh, Aaron Weiss, and Arjun Guha - University of Massachusetts at Amherst, USA . . . . . . . 416

Toward Compositional Verification of Interruptible OS Kernels and Device Drivers

Hao Chen, Xiongnan (Newman) Wu, Zhong Shao, Joshua Lockerman, and Ronghui Gu — Yale University, USA . . 431

Verified Peephole Optimizations for CompCert

Eric Mullen, Daryl Zuniga, Zachary Tatlock, and Dan Grossman — University of Washington, USA . . . . . . . 448

\section{Types II}

Just-in-Time Static Type Checking for Dynamic Languages

Brianna M. Ren and Jeffrey S. Foster - University of Maryland at College Park, USA . . . . . . . . . . . .

Types from Data: Making Structured Data First-Class Citizens in F\#

Tomas Petricek, Gustavo Guerra, and Don Syme - University of Cambridge, UK; Microsoft, UK; Microsoft Research,

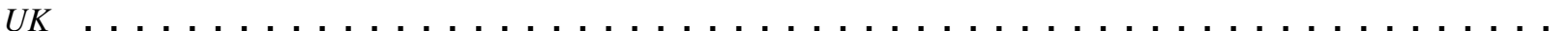

Automatically Learning Shape Specifications

He Zhu, Gustavo Petri, and Suresh Jagannathan — Purdue University, USA; University of Paris Diderot, France . . .

\section{Synthesis II}

Synthesizing Transformations on Hierarchically Structured Data

Navid Yaghmazadeh, Christian Klinger, Isil Dillig, and Swarat Chaudhuri - University of Texas at Austin, USA;

University of Freiburg, Germany; Rice University, USA . . . . . . . . . . . . . . . . . . . . . .

Program Synthesis from Polymorphic Refinement Types

Nadia Polikarpova, Ivan Kuraj, and Armando Solar-Lezama - Massachusetts Institute of Technology, USA . . . .

\section{Parallelism I}

Higher-Order and Tuple-Based Massively-Parallel Prefix Sums

Sepideh Maleki, Annie Yang, and Martin Burtscher — Texas State University, USA . . . . . . . . . . . .

A Distributed OpenCL Framework using Redundant Computation and Data Replication

Junghyun Kim, Gangwon Jo, Jaehoon Jung, Jungwon Kim, and Jaejin Lee — Seoul National University, South Korea

\section{Memory Management}

Idle Time Garbage Collection Scheduling

Ulan Degenbaev, Jochen Eisinger, Manfred Ernst, Ross McIlroy, and Hannes Payer — Google, Germany; Google, USA;

Google $U K \ldots \ldots$. . . . . . . . . . . . . . . . . . . . . . . . . . . . .

Assessing the Limits of Program-Specific Garbage Collection Performance

Nicholas Jacek, Meng-Chieh Chiu, Benjamin Marlin, and Eliot Moss — University of Massachusetts at Amherst, USA

\section{Verification II}

\section{Cardinalities and Universal Quantifiers for Verifying Parameterized Systems}

Klaus v. Gleissenthall, Nikolaj Bjørner, and Andrey Rybalchenko - TU Munich, USA; Microsoft Research, USA;

Microsoft Research, UK . . . . . . . . . . . . . . . . . . . . . . . . . . . .

Ivy: Safety Verification by Interactive Generalization

Oded Padon, Kenneth L. McMillan, Aurojit Panda, Mooly Sagiv, and Sharon Shoham - Tel Aviv University, Israel;

Microsoft Research, USA; University of California at Berkeley, USA . . . . . . . . . . . . . . . . . . .

\section{Security}

Precise, Dynamic Information Flow for Database-Backed Applications

Jean Yang, Travis Hance, Thomas H. Austin, Armando Solar-Lezama, Cormac Flanagan, and Stephen Chong — Carnegie Mellon University, USA; Dropbox, USA; San Jose State University, USA; Massachusetts Institute of Technology, USA;

University of California at Santa Cruz, USA; Harvard University, USA . . . . . . . . . . . . . . . . . . . 
End-to-End Verification of Information-Flow Security for $\mathbf{C}$ and Assembly Programs

David Costanzo, Zhong Shao, and Ronghui Gu - Yale University, USA . . . . . . . . . . . . . . . . . 648

A Design and Verification Methodology for Secure Isolated Regions

Rohit Sinha, Manuel Costa, Akash Lal, Nuno P. Lopes, Sriram Rajamani, Sanjit A. Seshia, and Kapil Vaswani -

University of California at Berkeley, USA; Microsoft Research, UK; Microsoft Research, India . . . . . . . . . . . .

\section{Parallelism II}

\section{Transactional Data Structure Libraries}

Alexander Spiegelman, Guy Golan-Gueta, and Idit Keidar - Technion, Israel; Yahoo Research, Israel . . . . . . . .

FlexVec: Auto-Vectorization for Irregular Loops

Sara S. Baghsorkhi, Nalini Vasudevan, and Youfeng Wu — Intel, USA; Google, USA . . . . . . . . . . .

Verified Lifting of Stencil Computations

Shoaib Kamil, Alvin Cheung, Shachar Itzhaky, and Armando Solar-Lezama - Adobe, USA; University of Washington,

USA; Massachusetts Institute of Technology, USA . . . . . . . . . . . . . . . . . . . . . . . .

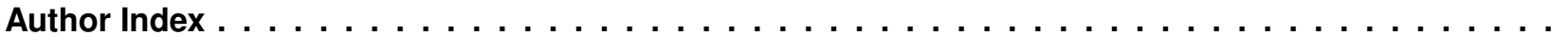

